\title{
A Priori Identifiability of Target-Mediated Drug Disposition Models and Approximations
}

\author{
Rena J. Eudy, ${ }^{1,2,4}$ Matthew M. Riggs, ${ }^{3}$ and Marc R. Gastonguay ${ }^{1,2,3}$
}

Received 23 March 2015; accepted 29 May 2015; published online 16 June 2015

\begin{abstract}
A priori identifiability of mathematical models assures that for a given input/output experiment, the parameter set has one unique solution within a defined space, independent of the experimental design. Many biologic therapeutics exhibit target-mediated drug disposition (TMDD), and use of the full compartmental model describing this system is well documented. In practice, estimation of the full parameter set for TMDD models, given real-world clinical data, is characterized by convergence difficulties and unstable solutions. Still, the formal assessment of the a priori identifiability of these systems has yet to be reported. The exact arithmetic rank (EAR) approach was used to test the a priori identifiability of a TMDD model as well as model approximations. The full TMDD and quasiequilibrium/rapid binding (QE/RB), quasi-steady state (QSS), and Michaelis-Menten (MM) approximations were fully identifiable, a priori, regardless of whether observations were taken from a single or multiple compartments. The results of these identifiability analyses indicated that the difficulty with TMDD model convergence, a posteriori, lies in the experimental design, not in the mathematical identifiability in the lack of samples from several compartments. Experiments can be tailored to resolve these structurally non-identifiable parameters, notwithstanding practical implementation challenges. This work highlights the importance of identifiability analyses, specifically how they can influence experimental design and selection of the appropriate model structure to describe a dynamic biological system.
\end{abstract}

KEY WORDS: approximation; a posteriori; a priori; identifiability; target-mediated.

\section{INTRODUCTION}

A priori global identifiability analysis is the process of determining if different combinations of parameter values lead to indistinguishable model output in terms of inherent model structure (1). A system is a priori globally identifiable (also known as structurally or mathematically identifiable) if all parameters have a unique solution within the full domain of the parameter space, given specific observation points within the system. A system is locally identifiable if a unique solution to a parameter is found within some neighborhood of that parameter (2). In contrast, a posteriori, also known as practical identifiability, is the quality of a system that determines whether or not parameters can be estimated

Electronic supplementary material The online version of this article (doi:10.1208/s12248-015-9795-8) contains supplementary material, which is available to authorized users.

\footnotetext{
${ }^{1}$ Metrum Institute, 2 Tunxis Road, Suite 112, Tariffville, Connecticut 06081, USA.

${ }^{2}$ Department of Biomedical Engineering, University of Connecticut, A.B.. Bronwell Building, Room 217, 260 Glenbrook Road, Unit 3247, Storrs, Connecticut 06269-3247, USA.

${ }^{3}$ Metrum Research Group, LLC, 2 Tunxis Road, Suite 112, Tariffville, Connecticut 06081, USA.

${ }^{4}$ To whom correspondence should be addressed. (e-mail: renae@metrumrg.com)
}

based on informativeness of an experimental design and resulting data. A priori identifiability is a requirement for a well-posed mathematical system and a prerequisite for parameter estimation because if identifiability is not achieved independent of experimental design conditions, the system will not be identifiable in practice. Determining which parameters in the system are not identifiable before running an experiment may save resources by informing experimental design. Unfortunately, this practice is underutilized because of the computational complexity involved in these analyses.

Establishing a priori, identifiability for the highly nonlinear models common in systems biology is especially nontrivial in cases when there are many more parameters than observables. A comparison of three approaches for evaluating identifiability for complex systems (two of these were focused on a priori) was recently published by Raue and colleagues (3) and forms the basis for the approaches considered in this analysis. Of these methods, the exact arithmetic rank (EAR) approach was the most robust and most easily implemented. The a priori identifiability of the widely used target-mediated drug disposition (TMDD) model, describing the dynamic system of antibodies binding to the target molecule (4), and approximations to this model has never been established. Gibiansky and Gibiansky also formulated extension of this model to include two-target TMDD (5). The model equations for the TMDD model represent amounts of free drug, target, the drug-target 
complex, and the transfer rates between these states (Fig. 1), and the extension includes dual targets and complexes (Fig. 2). Model approximations include quasi-equilibrium or rapid binding (QE/RB), quasi-steady state (QSS), and Michaelis-Menten (MM) (6). While approximations to the model make it possible for one to mathematically describe experimental data, the interpretation of these model parameter values can be vague or even inaccurate (6). Identifying system parameters that are not unique, a priori, indicates the need for re-parameterization or model simplification in advance of the parameter estimation step. A priori analyses may also assist in understanding which modifications of the experimental design are necessary to achieve meaningful parameter estimates.

\section{METHODS}

Of the three different approaches discussed by Raue and colleagues (3), the exact arithmetic rank (EAR) approach was chosen to evaluate parameter identifiability for TMDD models and their approximations. The EAR approach was developed by Karlsson and colleagues (7) and is designed to handle larger systems with more generally parameterized initial conditions. This algorithm constructs a symbolic form of the Jacobian matrix by way of generating a truncated power series expansion of partial derivatives of the output with respect to state variable $x(0)$ (the value of each compartment at its initial condition) and parameter $\theta$ and performs rank testing, the process of relating higher order derivatives to lower order derivatives and using the inverse function theorem, to determine local identifiability.

Single-target TMDD (Fig. 1) and QE/RB, QSS, and MM approximation models were tested first, followed by a TMDD model with two targets (Fig. 2). The input of each model was an intravenous (i.v.) infusion or subcutaneous (s.c.) dose. Whether or not the output scenario was plausible, all were included in the analysis for completeness and for use as a reference. To use EAR, the package "IdentifiabilityAnalysis" was loaded in Mathematica (v9.0). The differential equation set and initial conditions were assigned to variable deq. In this approach, the initial conditions must be supplied by the user,

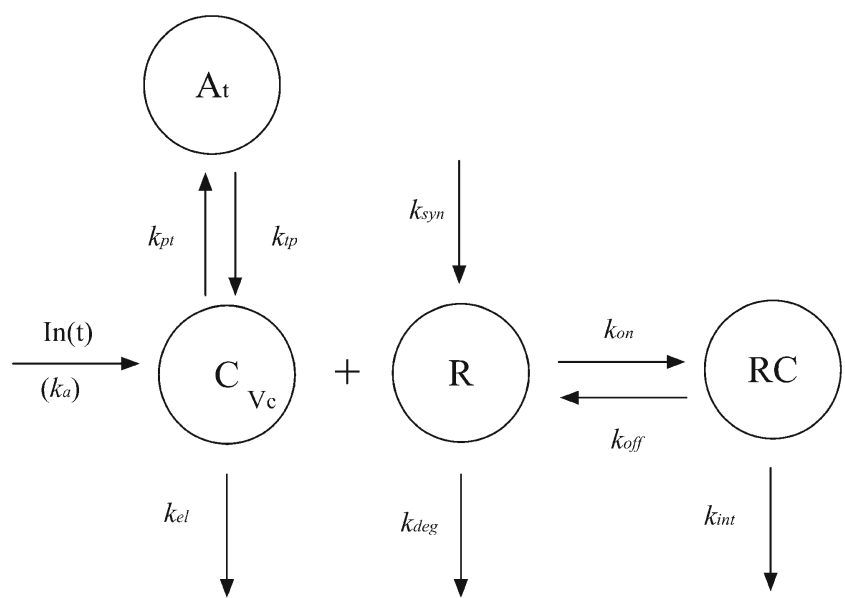

Fig. 1. TMDD model schematic. $C, R, R C$, and $A_{t}$ represent the drug concentration, target, drug-target complex, and peripheral compartments, respectively. $K s$ indicate time constants

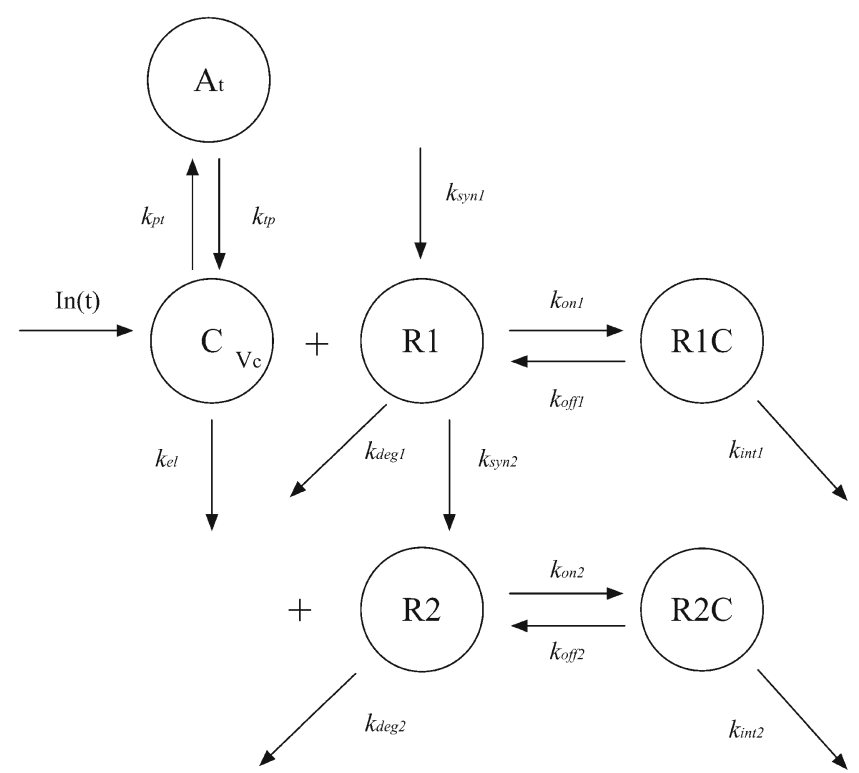

Fig. 2. Extension of the TMDD model to the two-target case. $C, R 1$, $R 2, R 1 C, R 2 C$ and $A_{t}$ represent the drug concentration, targets 1 and 2 , drug-target complexes 1 and 2, and peripheral compartments, respectively. Kss indicate time constants

even if they are unknown and set to zero, as they are required to construct the Jacobian. The arguments to the IdentifiabilityAnalysis function are simply $d e q$, the system outputs, a vector of variables, the independent variable $(t)$, and the input variable $(u)$. The vector of variables represents the parameters in the model but are supplied symbolically and assigned no values or bounds. If the results of the analysis are "false," the system is unidentifiable and the parameters without unique solutions can be identified with the call "NonIdentifiableParameters." Example code for a full TMDD model analysis is included in the Supplementary Materials.

\section{RESULTS}

Results for all model structures and output scenarios are shown in Tables I and II. The full single-target TMDD model was identifiable with any system output. It follows that the QE/RB, QSS, and MM are also identifiable with any output. Like the single-target TMDD, the 2target TMDD model structure was found to be identifiable under any output scenario.

\section{DISCUSSION}

The EAR application has been used to demonstrate $a$ priori identifiability of TMDD models and model approximations within scenarios when different outputs are available. The full TMDD model is a priori identifiable, even when only information about the drug (free or total) is available. This was not an obvious finding because a posteriori parameter estimates are often imprecise at best, and usually at least one parameter must be fixed in order for the model to converge. Gibiansky et al. show many examples of this in their 2009 publication (8). Often, it is the time scale of the drug measurements being much greater than that of the 


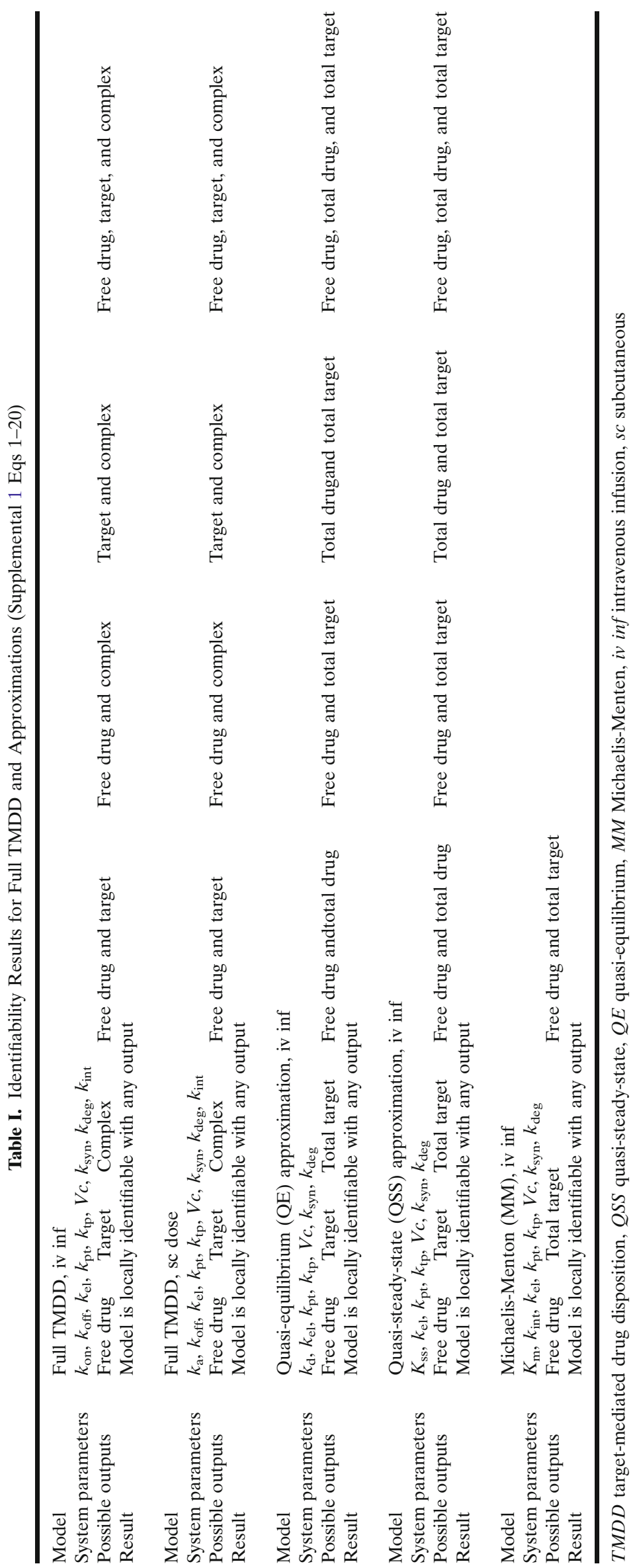


binding process that limits practical identifiability of kon and koff. The QE or QSS approximations are often used to avoid overparameterization. Selection between these approximations involves availability of target dynamics or pharmacodynamic data (9). Peletier and Gabrielson determined that specific regions of the drug concentration curve inform specific model parameters and parameter identifiability largely depends on the richness of data in each of these regions (10). These identifiability results confirm that all parameters in a full TMDD model can be uniquely estimated using a dataset with ideal sampling and no experimental noise. The full two-target TMDD system is also structurally identifiable under the same conditions.

Of the available approaches used for establishing structural identifiability of a system, the EAR algorithm was relied upon as the more robust of the approaches, specifically for approximations to the TMDD model when the model contains rational expressions that cannot easily be converted to polynomial. A limitation of EAR is that the algorithm cannot perform rank testing independent of initial conditions, and this limits the ability to establish unique solutions for some systems with initial conditions equal to zero. The differential identifiability of systems (DAISY) approach (11) was used as a first-pass attempt at establishing global a priori identifiability. Unfortunately, DAISY can only deal directly with pure polynomial forms for the differential equations, and the program did not accept the quadratic solution form for free drug concentration, $C$, that is used in the QE and QSS approximations. This shortcoming of DAISY is well documented $(3,12)$. Finally, the profile likelihood (PL) approach was also considered, but it is a data-based approach and not a specific analysis of a priori identifiability.

\section{CONCLUSION}

In the case of single-target TMDD models and approximations, all parameters are a priori identifiable, as is the full two-target TMDD model. These findings indicate that sampling times relative to the widely varying time scale of binding kinetics $v s$. distribution and elimination kinetics are the most likely culprit limiting a posteriori identifiability of TMDD model parameters. The principle of a priori identifiability is that a unique solution can be found under ideal sampling times and error-free experimental conditions. While noise-free systems do not exist, identifiability analyses are an important first step in model design. If parameters are found not to be structurally identifiable, they will never be $a$ posteriori identifiable when experimental noise and design limitations abound. As shown in Gibiansky and Gibiansky (13), it is possible to achieve good model fits without establishing identifiability of model parameters. In this same work, however, it was noted that resulting parameter estimates were not always accurate or reliable reflections of the proposed drug disposition mechanisms. Identifiability analyses, on the other hand, allow for meaningful interpretation of parameters under "real-world" conditions if model assumptions hold. Non-identifiability in these types of analyses can indicate the necessity for model simplification and show when more information is needed within a given experimental design to achieve a posteriori identifiability of 
all parameters. Recent advances of computational algorithms allow for easy and fast determination of structural identifiability of a biological system.

\section{REFERENCES}

1. Lamberton TO, Condon ND, Stow JL, Hamilton NA. On linear models and parameter identifiability in experimental biological systems. J Theor Biol. 2014;358:102-21. doi:10.1016/j.jtbi.2014.05.028.

2. Jacqueiz JA, Greif P. Numerical parameter identifiability and estimability: integrating identifiability, estimability, and optimal sampling design. Math Biosci. 1985;(77):201-27. doi:10.1016/00255564(85)90098-7.

3. Raue A, Karlsson J, Saccomani MP, Jirstrand M, Timmer J. Comparison of approaches for parameter identifiability analysis of biological systems. Bioinformatics. 25(15):1923-9. doi:10.1063/ 1.3528102 .

4. Mager DE, Jusko WJ. General pharmacokinetic model for drugs exhibiting target-mediated drug disposition. J Pharmacokinet Pharmacodyn. 2001;28(6):507-32.

5. Gibiansky L, Gibiansky E. Target-mediated drug disposition model for drugs that bind to more than one target. J Pharmacokinet Pharmacodyn. 2010;37(4):323-46. doi:10.1007/s10928010-9163-3.

6. Gibiansky L, Gibiansky E, Kakkar T, Ma P. Approximations of the target-mediated drug disposition model and identifiability of model parameters. J Pharmacokinet Pharmacodyn. 2008;35(5):573-91. doi:10.1007/s10928-008-9102-8.

7. Karlsson, J, Anguelova M, Jirstrand, M. An Efficient Method for Structural Identiability Analysis of Large Dynamic Systems. SYSID 2012: Proceedings of the 16th IFAC Symposium on System Identification; 2012 Jul 11-13; Brussels, Belgium. p. 941-946.

8. Gibiansky L, Gibiansky E. Target-mediated drug disposition model: approximations, identifiability of model parameters and applications to the population pharmacokinetic-pharmacodynamic modeling of biologics. Expert Opin Drug Metab Toxicol. 2009;5(7):803-12. doi:10.1517/17425250902992901.

9. Yan X, Mager DE, Krzyzanski W. Selection between MichaelisMenten and target-mediated drug disposition pharmacokinetic models. J Pharmacokinet Pharmacodyn. 2010;37(1):25-47. doi:10.1007/s10928-009-9142-8.

10. Peletier LA, Gabrielsson J. Dynamics of target-mediated drug disposition: characteristic profiles and parameter identification. $\mathbf{J}$ Pharmacokinet Pharmacodyn. 2012;39(5):429-51. doi:10.1007/ s10928-012-9260-6.

11. Saccomani MP, Bellu G. DAISY: an efficient tool to test global identifiability. Some case studies. Proceedings of the16th Mediterr Conf Control Autom. 2008 Jun 25-27; Ajaccio, France. p. 1723-8. doi:10.1109/MED.2008.4602152.

12. Chis O-T, Banga JR, Balsa-Canto E. Structural identifiability of systems biology models: a critical comparison of methods. PLoS ONE. 2011;6(11):e27755. doi:10.1371/journal.pone.0027755.

13. Gibiansky, L, Gibiansky E. Numerical Testing of Assumptions for Target-Mediated Drug Disposition (TMDD) Equations: Why Inexact Model Provides Satisfactory Description? PAGE 2014; Proceedings of Population Approach Group Europe Conference; 2014 Jun 11-14; Alicante, Spain. 